\title{
"Critical Language Learning" : le rôle de la littéracie et de la métacognition dans l'acquisition du français langue étrangère
}

\author{
Stéphanie Pellet \\ Wake Forest University \\ pelletsh@wfu.edu
}

\section{Introduction}

Qu'il s'agisse de l'enseignement des langues étrangères ou de l'éducation en général, un certain nombre d'études, réalisées aux Etats-Unis (Colby, Ehrlich, Beaumont, \& Stephens, 2003 ; Leithwood, Jantzi, \& Steinbach, 1999) ou en Europe (Lundwall, 2000; Cochinaux \& de Woot, 1995), ont souligné la responsabilité du corps enseignant quant à la formation d'étudiants capables de juger de façon critique, d'apprendre de façon indépendante, et d'être activement impliqués ou engagés dans le processus de leur formation. Cette orientation s'inscrit dans un objectif de compétition économique globale et est en grande partie le produit de l'avènement de la communication par ordinateur, qui donne lieu à de nouveaux modes de discours (discours multimédia entre autres), à de nouveaux supports pédagogiques, et en somme, à de nouveaux apprenants (« digital natives », Prensky, 2001). De part et d'autre de l'océan, une conviction de plus en plus forte s'impose parmi les pédagogues selon laquelle «the information society is going to change teaching methods by replacing the excessively passive teacher/pupil relationship with a new -and seemingly promising- interactive relationship » (White Paper, 1995 : 7). Les concepts pédagogiques clés sont ceux d'un apprentissage actif, collaboratif, et réfléchi (ou conscient). Parallèlement, plusieurs études, plus récentes celles-ci, soulignent l'importance grandissante accordée à l'écriture comme capacité critique dans une société dite d'information : l'enquête nationale de Lenart, Arafeh, Smith et Macgill (2008) sur les attitudes et croyances des adolescents américains ; ou celle de Belden, Russonello et Stewart (2007) sur l'opinion publique américaine concernant l'importance d'enseigner la rédaction à l'école.

La littéracie et la métacognition semblent donc constituer des outils offrant une capacité d'autonomie et d'apprentissage critique, ou réfléchi. Simultanément ces deux 'outils' apparaissent sous-exploités dans l'enseignement des langues étrangères, malgré des évolutions similaires vers l'autonomie de l'apprenant d'une langue seconde (Savignon, 2007) et vers une pédagogie dite 'active' (Niemi, 2002). Dans une ère reconnaissant les limites de 'méthodes' linguistiques successives (d'où la notion de 'postmethod' de Kumaravadivelu, 2002), les spécialistes en langues étrangères, qu'ils soient chercheurs ou enseignants (ou les deux), reconnaissent un malaise dans l'état actuel du cursus universitaire. Swaffar et Arens (2005) constatent par exemple que : "Seldom do departments discuss their programs in their entirety, that is, consider the ways in which their courses may or may not build toward consistent and realized achievement for majors and nonmajors alike and prepare their students to use language effectively in the real world. » (p. xi), tandis que Stivale note la nécessité pour l'enseignement du français d'entreprendre « a multifaceted reflection on pedagogical practices in a particular disciplinary field and, by extension, in adjacent domains - literary, cultural, and foreign language studies » (2004:2).

Ce malaise est accentué par le fait que le contexte d'enseignement du français langue seconde présente un décalage entre recherches et développements théoriques et mises en application dans la salle de classe (Barbazan, 2008; Elalouf, 2008). D’une part, le développement de la linguistique interactionnelle (Mondada, 2008) appelle des pédagogies langagières plus ancrées dans la réalité des situations communicatives et leurs objectifs; une approche critique des études du langage, "Critical Language Awareness », a mis en évidence les discours dominants et a donné lieu à de nouvelles sous-branches en linguistique (analyse critique du discours, linguistique appliquée critique) et à la notion de littéracie (Barré-De Miniac, Brissaud, \& Rispail, 2004; Swaffar \& Arens, 2005); et finalement les théories 
constructives d'apprentissage des langues, fondées sur l'aspect fondamentalement social du processus d'apprentissage (Bruner, Vygotsky, Dewey) et élaborées à partir de l'approche collaborative, créent la possibilité d'un rôle plus actif et autonome pour l'apprenant. D'autre part, linguistique interactionnelle, « Critical Language Awareness », littéracie (critique), et approches constructives à l'apprentissage ont en commun d'être en-deça de la didactique des langues étrangères. Critical Language Awareness et littéracie, par exemple, ont longtemps été le mode privilégié d'approches à la langue anglaise, (à travers notamment le concept de "World Englishes »), et la notion de littéracie elle-même a longtemps été exclusivement anglophone. La langue française ne s'est emparée de cette notion que récemment (BarréDe Miniac, Brissaud \& Rispail, 2004), et essentiellement dans le but d'améliorer le rapport à la lecture et à l'écriture dans la langue natale. La littéracie pour les apprenants langue seconde a cependant fait l'objet de quelques études récentes : trois se situent dans le cadre spécifique des apprenants immersés dans la communauté linguistique seconde (Boyd, Brock \& Rozendal, 2004 ; Koda \& Zehler, 2008), en particulier les hispanophones aux États-Unis (August \& Shanahan, 2006); elles approchent donc la littéracie plus dans le sens traditionnel des compétences d'écriture et de lecture (comprendre le sens d'un texte; savoir écrire 'correctement'). Trois études (Byrnes \& Sprang, 2003 ; Kern, 2003 ; Swaffar, 2003) explorent conjointement la littéracie en langue étrangère pour les apprenants avancés et se rapprochent donc plus précisément du contexte de la présente étude. Ces études prennent toutes trois comme point de départ le constat suivant : les compétences insuffisantes des étudiants dits 'avancés' sont le reflet d'une part du décalage existant entre les développements théoriques et mises en application qui viennent d'être mentionnées, et d'autre part du manque d'un programme didactique (cursus) en langues étrangères qui soit cohérent et intégré.

La problématique qui vient d'être sommairement présentée inspire deux évolutions pratiques pour l'enseignement du français : d'une part, celle d'une approche pédagogique critique dont les objectifs sont de développer chez les apprenants des pratiques de réflexion plus indépendante et critique pour 'lire' textes, situations et évènements inter-culturels en prenant en compte le soi, l'autre, le discours dominant, et les voix minoritaires; et d'autre part, celle d'un développement de pratiques métacognitives liées au milieu académique (techniques de recherche, d'écriture, et de lecture de textes académiques) mais dont la portée s'étend au-delà de l'espace universitaire -la sphère professionnelle demandant également la capacité d'écrire, de lire de façon critique, et d'identifier des sources d'information. L'objectif de cette communication est donc de proposer un enseignement des langues encourageant une réflexion métacognitive et une connaissance langagière plus critique à travers la littéracie appliquée aux pratiques discursives académques; et d'appuyer cette didactique langagière responsable sur une description des facteurs qui créent à la fois le contexte la rendant nécessaire et les conditions la rendant possible. Un certain nombre de stratégies mises en place dans le cadre de deux cours pour apprenants avancés du français langue étrangère (Langage et Société, Le français moderne) permettent d'envisager une mise en application concrète. L'article termine avec un bref aperçu des 'nouvelles littéracies' et des leçons qu'elles peuvent suggérer pour une littéracie L2.

\section{Linguistique interactionnelle et didactique du français langue étrangère : un écart à combler}

\subsection{La variation et la métalangue absentes du curriculum}

Alors même que la vision qui sous-tend les programmes de langues étrangères est en train d'élargir le modèle traditionnel « littérature, culture et langue » afin de mieux incarner la complexité du langage dans ses rôles interactionnels (Swaffar \& Arens, 2005), dans la pratique, les étudiants en français langue étrangère continuent de n'avoir accès qu'à une représentation partielle du système de la langue, centrée autour d'une grammaire relativement abstraite, dépouillée de sa réalité et complexité discursive.

Pourtant sur le plan théorique, la didactique des langues profite depuis plusieurs décennies des recherches fondées sur l'usage de la langue, ce que Mondada (2008) appelle la linguistique interactionnelle. Celle-ci met en avant les « articulations entre syntaxe et pragmatique, entre propriétés structurelles de la langue et 
ses usages en société, entre contraintes formelles et fonctionnelles » (Mondada, 2008: 881). Ainsi les recherches fondées sur des corpus de données réelles, l'analyse du discours, de la conversation, les études de variation sociolinguistique et les travaux sur la structure de l'information ont-elles mis à jour les différences existant entre langue standard et langue parlée (voir par exemple les travaux de BlancheBeneviste ou bien ceux de Valdman sur la norme pédagogique). Elles favorisent une grammaire contextualisée, tant au point de vue social que situationnel: cette «grammaire du discours» est naturellement complétée par une " grammaire pédagogique ", c'est-à-dire une grammaire orientée vers les apprenants (Odlin, 1994). Katz et Blyth (2007) proposent une " grammaire instructionnelle », celle-ci orientée vers ces enseignants (en particulier les étudiants-enseignants comme les doctorants), qui faute d'avoir eux-mêmes reçu un enseignement sur les liens entre grammaire et discours ou sur la façon de présenter ces aspects à leurs étudiants, reproduisent le schéma traditionnel d'une grammaire séparée de l'acte communicatif. Katz et Blyth offrent donc une relecture d'éléments de grammaire française à la lumière des intentions des locuteurs et de la situation discursive, et proposent des activités pédagogiques permettant aux apprenants de comprendre les mécanismes pragmatiques derrière les structures de la langue. Cependant, comme le soulignent ces deux auteurs, un écart important demeure entre les recherches en acquisition d'une langue seconde et les implications pédagogiques. Les jeunes enseignants manquent en particulier d'une métalangue leur permettant d'expliquer les points de grammaire dépendants du discours, ainsi que de la conscience du rôle des facteurs sociolinguistiques et pragmatiques sur les formes grammaticales (Katz \& Blyth, 2007: 4). Selon Barbizan, «le FLE a des difficultés à intégrer en un système cohérent des notions énonciatives ou textuelles encore trop parcellisées, et qui ne sont pas reliées aux connaissances grammaticales traditionnelles » (2008:383), tandis qu'Elalouf (2008) parle de « résistances ».

Le vide pédagogique touchant les mécanismes linguistiques qui sous-tendent les intentions du locuteur, en particulier du point de vue de la dimension perlocutoire des énoncés, s'explique de diverses façons. Le premier point est la croyance générale selon laquelle les connaissances métalinguistiques ne sont pas à la portée des étudiants en langues étrangères, puisqu'elles requièrent premièrement une connaissance basique en linguistique. Pourtant, comme le font remarquer Rose et Kasper (2001), la plupart des aspects pragmatiques sont 'enseignables' bien que les stratégies pédagogiques doivent être adaptées en fonction des objectifs, des étudiants et du contexte institutionnel et socioculturel (Rose \& Kasper, 2001: 8). Le deuxième point est un héritage de la perception de la dimension (socio)pragmatique comme «waste basket » de la linguistique. Dans cette perspective, la pragmatique et la sociolinguistique ne font pas intrinsèquement partie d'un cursus en langue étrangère. Les impératifs dominant la didactique sont (toujours) les traits formels de la langue, largement appuyés sur la grammaire. Troisièmement, la pédagogie prédominante reste fondée sur une représentation homogène de la langue, excluant donc la variation dans toutes ses formes (sociale, pragmatique). C'est l'argument soutenu par exemple par Train $(2003$; 2007) pour qui ce n'est pas la variation qui est le problème, mais bien plutôt la standardisation (2007: 240). Sociolinguistique et pragmatique (c'est-à-dire variation) présentent des «problèmes pédagogiques » (Train, 2003, 2007) dans la didactique des langues étrangères précisément parce que ces domaines privilégient les usages de la langue et non la norme. Train expose «la notion de Langue standard native (NSL) comme un ensemble d'idéologies langagières qui, par le processus complexe de la standardisation, arrive à définir les 'réalités' culturelles, linguistiques, et identitaires pour les apprenants et les enseignants du français aussi bien que pour les locuteurs dits natifs que pour les non-natifs » (2007: 238). Un des problèmes que Train met ainsi en lumière est l'aspect statique de "réalités définies », annihilant l'aspect dynamique de la langue. Or langue, communauté linguistique et environnement ne sont jamais stables.

\section{2 Émergence d'une didactique centrée sur l'apprenant : autonomie, identité, participation}

En donnant naissance à une perspective de la langue comme un outil (social) de communication, la linguistique interactionnelle a aussi participé à une redéfinition de l'apprenant des langues étrangères. L'apprentissage a ainsi pu passer d'une approche centrée sur l'enseignant (teacher-centered approach) à 
une approche centrée sur l'apprenant (student-centered approach), l'enseignement de la langue visant à développer des compétences communicatives (à travers notamment l'approche dite "Communicative Language Teaching »), c'est-à-dire, pouvoir utiliser la langue étrangère dans des situations concrètes, quotidiennes. Malgré des erreurs interprétatives et des critiques (en particulier lié au déficit grammatical qu'elle a engendré), cette approche a permis un déplacement de l'attention de l'enseignement sur l'apprenant, en parallèle à une linguistique interactionnelle déplaçant l'attention du système de la langue aux (intentions des) locuteurs et interlocuteurs. La notion d'autonomie de l'apprenant (Savignon, 2007) a gagné du terrain en didactique des langues étrangères, supportée par un discours revendiquant la construction d'une identité propre par l'apprenant (Kramsch, 1997) : cette identité n'est plus uniquement modelée sur le locuteur natif, puisque ce concept ne capture pas la complexité sociolinguistique des usages réels de la langue. L'apprenant reçoit donc un rôle actif et participatif par rapport à son propre apprentissage. Une didactique émerge donc, basée sur une habilitation (empowerment) de l'apprenant et visant une acquisition pertinente, pragmatique (au sens large) de la langue et une sensibilité interculturelle (Pellet, 2010a). L'objectif d'autonomie de l'apprenant se situe également au niveau de l'évaluation « authentique » (Kohonen, 1998) (par opposition à l'examen au format standard) et dont le but est d'attirer l'attention de l'apprenant sur l'état (trajectoire) de son apprentissage par l'intermédiaire notamment d'entretiens oraux, de portfolios et de projets. Ce type d'évaluation s'inscrit aussi dans une logique de réflexion de l'apprenant sur ce qu'apprendre veut dire. Si l'apprenant gagne une certaine autonomie et une identité propre, Maxim (2003) met cependant la profession en garde contre le danger d'un centrisme sur l'apprenant, affirmant qu'une approche aidant les étudiants à développer leur propre voix « represents a strongly individualistic rather than a social understanding of language because the individually created expressions might be linguistically accurate, but they only become truly meaningful if they are situated in a social context and accepted by a discourse community » (p. 197). Cette mise en garde, valide, ne s'oppose pas à un rôle participatif des étudiants dans leur accès aux connaissances mais elle attire l'attention sur la nécessité de donner une voix, une identité aux apprenants qui soit située socialement, comme il l'est discuté dans la section précédente.

En parallèle, les théories socio-constructivistes (Vygotsky, Dewey) ont participé au transfert d'un focus de l'enseignement sur l'apprenant, plutôt que sur un contenu pré-déterminé et sur l'autorité de l'enseignant. Ce faisant, les approches dites «student-centered» changent non seulement la nature des interactions entre apprenants et enseignant, mais aussi les conditions d'accès au savoir. Les approches constructives créent des conditions plus directes d'accès au savoir en particulier à travers l'apprentissage collaboratif entre étudiants (Bosworth \& Hamilton, 1994; Bruffee, 1995), et donc la possibilité d'engagement actif des apprenants par rapport à ce savoir (Bonwell \& Eisen, 1991; Meyers \& Jones, 1993). Elles créent également la possibilité d'ouvrir le domaine de la recherche à un niveau plus précoce d'étude.

\subsection{Langue et compétences dans le cursus du français langue étrangère}

Il n'y a aucun doute que le passage à une didactique des langues étrangères axée sur la communication et l'interaction a représenté un progrès, mais ce faisant, un autre rôle essentiel de la langue seconde a été oblitéré : celui qu'il joue dans l'acquisition même des connaissances. Cela a été la contribution en particulier de Bruner à la psychologie de l'apprentissage, puisqu'il a souligné le rôle des compétences langagières dans une approche aux connaissances axée sur une découverte guidée. Pour Bruner, "[t]eaching is vastly facilitated by the medium of language, which ends by being not only the medium for exchange but the instrument that the learner can then use himself in bringing order into the environment. The nature of language and the functions it serves must be part of any theory of cognitive development" (1968 : 6). Dans sa chronique de l'écriture académique, Russel affirme ainsi que «Bruner's theorizing showed the possibility of consciously, systematically using writing as a tool for learning in a specific disciplinary context, rather than as a generalized skill» (2002: 247). La question des compétences métalinguistiques a été bien comprise par les éducateurs et les enseignants-chercheurs spécialistes de l'écriture académique : dans leur contribution à un ouvrage élaborant sur ce que « Preparing teachers for a changing world» signifie, Valdès, Bunch, Snow et Lee (2005) insistent sur l'importance de développer 
les compétences langagières spécifiquement académiques des étudiants futurs enseignants qui plus souvent font face à des enseignants qui ne sont pas conscients de leur propre usage de la langue. Le développement de ces compétences s'appuye sur une prise de conscience de la variation des usages de la langue selon les différents contextes sociaux, le milieu académique représentant un contexte social particulier. Si cela est vrai pour l'apprentissage des disciplines en général, cela n'en est pas moins vrai pour l'apprentissage de la langue étrangère. La langue étrangère n'est donc pas seulement l'objet d'études (la discipline), mais également le moyen par lequel l'étudiant apprend, et il est moins clair que ce point soit saillant dans la conscience pédagogique des enseignants du français langue étrangère (ou des autres langues), et surtout dans la conscience de ceux qui forment ces enseignants.

L'organisation didactique même des départements universitaires américains en langues étrangères, par leur haut degré de cloisonnement à de multiples niveaux, participe au clivage entre la langue comme objet d'étude et comme outil d'étude. Deux niveaux de clivage sont à souligner : cloisonnement (en anglais 'bifurcation' pour Byrnes et Maxim, 2003, et leurs collaborateurs) tout d'abord entre les (deux) années de formation linguistique initiale, dont l'objectif est de produire des étudiants ayant une compétence essentiellement orale, et les cours plus avancés dont le sujet n'est plus la langue elle-même, mais un sujet lié à la langue (culture, littérature, civilisation, linguistique). Dans ce schéma, «because of learners' presumed mastery of the language at this stage of instruction, very little overt attention is devoted to comprehending and navigating the discursive and rhetorical patterns that are associated with a range of written and oral genres » (Maxim, $2003: 181$ ). Le deuxième type de cloisonnement provient de la spécialisation des cours avancés : les cours en littérature, culture / civilisation, et linguistique ont chacun leurs objectifs précis en termes de contenu. Mais la conception autonomiste de ces cours font que leurs objectifs ne laissent que peu (ou pas) de place à un raffinement des connaissances et compétences langagières.

D'autre part, l'objectif initial de compétence interactionnelle de l'approche communicative, en favorisant l'expression orale, divertit l'attention de la compétence 'textuelle' (Maxim, 2003 : 196) nécessaire pour accéder à un niveau supérieur de compétence. Thorne et Reinhardt (2008) soulignent de plus que l'importance et le prestige associés aux cours de littérature (plutôt qu'aux cours de langue) font que « there are relatively few language-focused pedagogical texts or materials that focus on mastery of subtle features of interactive spoken and written discourse » (p. 559).

La conception même du cursus conduit donc à une opacité 'discursive et rhétorique' de la langue pour les apprenants, opacité renforcée par le fait qu'elle constitue au contraire une transparence pour les enseignants (Valdès et al., 2005); et à un clivage entre langue objet et langue outil. Une approche basée sur la littéracie permet de souligner les interactions qui existent « among form, context, and function in all [the students'] uses of language -whether they are speaking, listening, reading, or writing » (Kern, 2003 : 7). De plus, l'usage de la langue devenant un objet d'analyse et de reflection, «a focus on literacy removes the artificial separation of skills and content » (ibidem, p. 7) et permet de situer cet usage dans son contexte culturel et social.

\section{Littéracie et pratiques métacognitives}

\section{1 Émergence d'une littéracie L2}

La littéracie appliquée à la langue étrangère est une approche qui a été proposée en particulier par Kern (2000) dans le but de remédier à une opacité socio-culturelle des pratiques discursives. Cette approche est en continuité avec les travaux du 'New London Group' (1996), un ensemble de chercheurs anglophones (Australie, États-Unis, Angleterre) ${ }^{1}$, sur une littéracie plus élaborée que le modèle traditionnel de compétences basiques de lecture et d'écriture (essentiellement associé au cursus primaire). Le modèle proposé est issu d'une prise de conscience de la complexité sociale des interactions dans une société évoluant vers la globalisation (d'où leur notion de 'multilittéracie'). Il vise une analyse textuelle prenant en compte les aspects socio-culturels du discours pour une compétence pratique et critique, d'où les 
quatres éléments constitutifs de 'pratique située' (le social et le culturel), d' 'instruction explicite' dans un 'cadre critique' conduisant à une 'pratique transformée'. Ces quatres aspects forment une fondation essentielle pour une pédagogie visant une appropriation discursive par l'apprenant: la littéracie, accordant une large place à la métalangue (et par tant la métacognition), constitue donc bien un outil.

A l'ouvrage initial de Kern (2000) se sont ajoutées trois études qui forment la première partie d'une réflexion sur le cursus en langues étrangères en place dans les universités américaines, qui ne semble pas être en mesure de 'produire' des compétences réelles chez leurs étudiants avancés (Byrnes \& Maxim, $2003)$ : en réponse, Kern, Swaffar, et en collaboration Byrnes et Sprang proposent la «literacy as a conceptual framework for collegiate advanced learning ». Ces trois recherches posent les bases d'une pédagogie fondée sur la littéracie «as an organizing principle to design language curricula that problematize the linguistic, cognitive, and social relationships that link readers, writers, texts, and culture » (Kern, $2003: 2$ ), principe qui doit adresser le problème de la 'bifurcation' et du vide du cursus envers les compétences langagières qui s'ensuit. Kern suggère que la littéracie peut donner une dimension nouvelle à la narration (type de discours) et aux projets de recherche thématiques (par exemple, le colonialisme) ; Swaffar propose le précis comme genre servant de modèle pour l'appropriation d'autres genres liés à l'analyse littéraire (le roman, le film...), et permettant notamment de mettre en avant les notions de discours formel / informel, privé / public; tandis que Byrnes et Sprang proposent une « approche cognitive basée sur le genre », détaillant cette approche à travers les exemples de la narration et du discours politique (un type de discours public).

Les trois études ont en commun d'être orientées sur la notion de 'genre' faisant le lien avec les exercices littéraires des cours traditionnels et sur les types de discours communs dans une conception de participation à la vie sociale au-delà de la vie quotidienne. Elles sont donc une contribution importante par le regard nouveau qu'elles offrent sur le rôle de l'analyse textuelle pour une compétence communicative plus sophistiquée. La littéracie spécifiquement orientée vers l'appropriation du discours académique reste cependant largement négligée dans les cours de langues étrangères. C'est de cette littéracie particulière dont il est traité ici.

\subsection{Définir les enjeux pédagogiques}

La littéracie joue un rôle critique dans la mesure où « tout apprentissage d'une écriture se transforme en une activité métalinguistique qui implique la capacité à écouter sa propre langue, à l'analyser, avant même de la représenter. L'écriture est donc, de ce point de vue, un puissant outil cognitif » (Jaffré, 2004 : 36-37, citant les travaux d'Olson, 1999). Magnotto Neff et Whithaus (2008) soulignent également le rôle essentiel de l'écriture au sein de l'apprentissage : «[w]riting is a way of thinking, learning, and discovering; writing leads to increased learning and better retention; and writing helps students master the language and strategies of particular disciplines and become actively connected to those disciplines » (p. 3). La littéracie permet ainsi de « comprendre la complexité des pratiques d'écrit dans leur diversité et leurs interrelations » (Barré-De Miniac, Brissaud \& Rispail, 2004: 7) afin de pouvoir développer une compétence envers le texte, qu'il s'agisse de le lire (interprétation) ou de le produire. L'appel récent à un cursus en langues étrangères fondé sur de «multiples littéracies» (Swaffar \& Arens, 2005) élargit la notion de «texte» non seulement au niveau du genre (textes sonores, audiovisuels, imprimés, électroniques, artistiques et graphiques) (Anderson, Carr, Lewis, Salvatori \& Turnbull, 2007) mais plus spécifiquement au niveau de l'engagement des apprenants avec le texte (littéraire ou autre).

Cet appel est en contraste avec le constat des insuffisances au niveau des capacités en écriture probablement commun à tout département de langues étrangères (et de fait dans beaucoup d'autres) et qui pourrait bien être lié au fait qu'il existe peu de tentatives d'établir un modèle théorique d'écriture en langue seconde (Macaro, $2003: 221$ ). Le modèle le plus fréquemment cité date du début des années quatre-vingts (Flower \& Hayes, 1981). Dans le cas du français langue seconde, Katz et Blyth (2007) rapportent les plaintes fréquentes des enseignants vis-à-vis de leurs étudiants incapables d'écrire ou d'épeler et dont la maîtrise en grammaire est « atroce» (2007: 10). Weber-Fève (2009) cite un rapport en 2004 qui fait état des déficiences en matière d'écriture de jeunes diplômés d'universités américaines et de 
lycées dans leur activité professionnelle. Par ailleurs, Kamler et Thompson (2004) notent que ces difficultés sont également présentes chez les étudiants poursuivant une carrière académique (doctorat), et rappellent que de nombreuses études font état de cette situation à divers niveaux : difficultés d'achever la dissertation doctorale liées à l'angoisse de la production écrite (par opposition à l'étape de recherche) et au manque de continuité entre processus de recherche et d'écriture. Plusieurs recherches soulignent la tendance des enseignants d'appréhender l'écriture comme un produit plutôt que comme un processus, bien que le processus d'écriture comme approche pédagogique ait déjà été suggéré à plusieurs reprises (Macaro, 2003 : 226). C'est le cas notamment des mouvements «Writing Across the Curriculum » et «Writing Across Disciplines », nés dans les années quatre-vingts, dans le but d'intégrer un contenu pluridisciplinaire avec les techniques d'écriture pour une approche plus critique à la connaissance (Russel, 2002 ; Sorcinelli \& Elbow, 1997).

La lecture et l'écriture tendent aussi à être approchées soit comme deux compétences distinctes (Way, Joiner \& Seaman, 2000, cité dans Macaro, 2003 : 224), soit au contraire comme si la première engendrait 'spontanément' le développement de la seconde (Pereira, 2004), attitude que saisit la formule de Russel, « reading goes in and writing comes out» (2002: 243). Le paradoxe qui en ressort est que les étudiants doivent constamment lire et produire des textes dans le cadre de leurs cours, mais qu'il existe peu de gouvernance, celle-ci étant essentiellement assignée à la langue natale (l'anglais pour les étudiants anglophones, le français pour les étudiants francophones, etc...). Le constat quotidien par les professeurs de langues étrangères selon lequel les étudiants « ne savent pas écrire » et « attendent la dernière minute » pour rédiger un travail (composition ou recherche) coexiste avec une assomption générale selon laquelle entreprendre des études supérieures suppose une capacité littéraire minimale, et que les étudiants ayant gagné accès aux études universitaires ont par définition déjà «plus ou moins » acquis cette capacité. En réalité, les étudiants ont probablement acquis une certaine connaissance (avec une variation individuelle marquée) par rapport à l'exercice de composition, c'est-à-dire la conscience d'éléments minimaux requis : une organisation (structure logique), et un argument étayé ; des stratégies organisatrices (par exemple, marquer un lien entre deux paragraphes ou sections) ; une bibiographie (à valeur d'alibi ?). Mais cela a peu à voir avec la recherche académique, qui requiert selon l'expression anglaise consacrée « breath and depth » (Gale, 1996), ce qui amène Slevin (2007) à se plaindre que la production écrite des étudiants «went on to become institutionalized in its weakest, least intellectual form and meaning » (2007: 208).

De plus, cette compétence n'est pas nécessairement transférée à la langue seconde, un trait connu de la recherche en acquisition d'une langue étrangère. Le manque de transfert de compétence entre L1 et L2 est documenté en ce qui concerne la lecture sous le nom d'hypothèse de court-circuit (Clarke, 1979, cité dans Macaro, 2003 : 127). Macaro la résume avec la formule suivante : «L2 reading ability $=\mathrm{L} 1$ reading ability + L2 language proficiency » (2003 : 129). En d'autres termes, une acquisition 'modulaire' de la langue n'est pas possible. En ce qui concerne les immigrants apprenant l'anglais, August et Shanahan suggèrent ainsi que « the components of English-language proficiency that are linked to English reading proficiency are English vocabulary knowledge, listening comprehension, syntactic skills, and the ability to handle metalinguistic aspects of language (such as providing definitions of words) » (2006:64). De plus, « the acquisition of proficient writing skills entails good spelling skills, metacognitive skills such as audience awareness, and familiarity with and opportunities to practice writing different text genres » (ibidem, p.65). Une approche intégrative, mettant en relation lecture, écriture et métacognition, paraît nécessaire. Or les approches pédagogiques envers la lecture et l'écriture semblent plutôt fragmentées.

\subsection{Fragmentation de l'écriture et de la lecture}

La carence métacognitive observée sur le plan structural de la langue a son pendant dans le domaine littéraire. Les professeurs responsables de cours de français langue étrangère orientés sur la littérature (au sens large, incluant les cours sur le cinéma et la culture française et francophone), accordent une grande importance à la composition ou l'essai dans leurs cours, mais les étudiants ont-ils accès à une métaconnaissance à travers l'étude d'articles sur les auteurs et les œuvres (littéraires, cinématographiques ou autres) discutés en classe? Au niveau débutant, l'écriture porte en particulier sur la description (souvent 
liée à des situations mettant en avant les expériences personnelles) et représente essentiellement une pratique dont le but consiste à développer la maîtrise des connaissances formelles de la langue, une pratique dénoncée comme dénuée de valeur ou contenu communicatif, un exercice abstrait purement académique (Terry, 1989). C'est l'exemple typique des cours de composition et / ou de grammaire avancée. La narration et l'argumentation marquent la progression des activités écrites en parallèle à une progression des compétences langagières. L'essai critique est généralement réservé au cours de littérature et culture (y compris les cours sur le cinéma) mais les attentes des enseignants sont limitées, comme l'indiquent les commentaires suivants d'enseignants (universitaires) du français interrogés. Le premier commentaire décrit les projets écrits pour une série de cours de littérature du niveau intermédiaire au niveau avancé :

«My students write what I call a "commentaire de texte." I give them a list of topics (and I also invite them to discuss an original topic with me) and they write an essay with limited or no secondary sources. [...], these papers are 2-3 pages and 4 to 5 in the upper levels. In French [numéro du cours] and above, I usually have my students do an oral presentation "that will enhance or complement our discussion of the text we are currently studying." This is where the research component comes in. I ask the students to be creative with this project ---most of them do some sort of power point presentation (some include images, clips, music etc.) As for documenting their sources, they just include a "bibliographical slide." I meet with each group (students may work alone or in pairs) before they do this project, and we go over what type of research would be best --internet, sources in the library, --it often depends on the topic. I also spend a lot of time helping them with grammar! »

Le deuxième commentaire situe l'écriture dans le cours de cinéma francophone :

«In my cinema class, the students have a "project" that consists of four parts -- part one is a paper (compte rendu) on one film (10\%); part two is an outline of their project with several components such as synopses of three films (including the one they analyzed for part one), a bibliography of outside sources, a filmography of director(s), etc. (10\%); part three is a PowerPoint presentation with handouts on the project they outlined in part three; part four is a final paper with outside sources. The outside sources I require are somewhat limited -- I ask that they use books (if there are any on the subject) and journal articles from Cahiers du cinéma and others, and I ask that they limit their use of online sources. They usually use approximately five-seven sources. »

Ces commentaires isolés ont valeur anecdotale. Cependant ils illustrent les tendances évoquées par d'autres recherches (Macaro, 2003), et font écho à l'affirmation de Slevin selon lequel «literature teachers often require a great deal of writing, but they have not generally transformed student writing into cultural objects for critical interpretation » (2007: 209). Dans son contexte original, cette affirmation concerne la langue anglaise (langue maternelle), mais il est aisé de voir une similarité en ce qui concerne l'écriture dans le cours de langue étrangère.

Face aux activités écrites traditionnelles, l'enseignant se trouve souvent soumis au choix suivant : soit il accorde priorité à la 'production' de plusieurs activités soumises une seule fois et dont les corrections font partie de la note finale reçue pour chaque activité (par exemple, 4 compositions durant le terme, en plus d'autres écrits plus courts et des examens); soit il accorde la priorité au processus de révision, et l'étudiant doit resoumettre, une fois ou davantage, son travail écrit. Dans le premier cas, l'étudiant risque de ne pas intégrer les commentaires de l'enseignant : même s'il / elle les lit, c'est plutôt pour accepter la note reçue (Macaro, 2003 : 239), et souvent les enseignants commentent les productions écrites dans le but de justifier la note. Les commentaires ont donc une faible valeur pédagogique. Dans la situation alternative, les corrections tendent à concerner la forme, sur laquelle il est plus facile de commenter. Les commentaires sur le contenu sont également problématiques. D'une part, ils représentent un investissement relativement lourd pour l'enseignant qui doit équilibrer un feedback suffisamment informatif tout en évitant de 'mâcher' le travail pour l'étudiant. D'autre part, les étudiants ne comprennent pas toujours les commentaires qu'ils reçoivent (Elola, $2008: 52$ ), d'autant que souvent ces commentaires sont sous forme de fragments (Macaro, 2003 : 240). Les évaluations des travaux écrits privilégient donc 
l'aspect 'sommatif' au détriment de l'aspect 'continu' (c'est-à-dire permettant l'évaluation de la progression) (Kohonen, 1998) et ont donc peu de valeur pédagogique.

La lecture débutante, quant à elle, favorise une approche dans laquelle l'apprenant est encouragé à prendre le texte 'pour argent comptant' de par le focus sur la compréhension 'littérale' du texte comme première compétence requise. Macaro évoque par ailleurs une « invisibilité relative de la capacité à lire » (2003 : 118), c'est-à-dire qu'il est plus facile d'évaluer la qualité d'un texte écrit par un apprenant que celle de la lecture par ce même apprenant. Les activités typiques vérifiant la comprehension manquent de substance si bien que "true/false, multiple choice and other similar task types are all very well for keeping the students busy but they do not get at the processes involved in their reading » (Macaro, 2003 : 151). Aux niveaux intermédiaire et avancé, une approche probablement fréquente consiste à « faire lire » une œuvre ou divers extraits par les étudiants. Le cours fournit une contextualisation et une lecture critique par le professeur. Puis les étudiants sont amenés à produire un essai mettant en avant un texte (littéraire), les notes de cours dûment prises, et quelques sources secondes, officiellement présentées dans la note bibliographique. Les étudiants sont bien conscients que ces références bibliographiques doivent être présentes et qu'elles légitiment l'essai. Tout comme les professeurs sont bien conscients que ces références n'ont souvent été que peu consultées. Y a-t-il eu, dans la plupart des cas, une discussion sur des articles de référence, que ce soit sur le fond ou sur le contenu ? Si la réponse est non, c'est peu surprenant dans la conception traditionnelle de l'enseignement : les articles de référence sont réservés aux spécialistes (les enseignants chercheurs); et l'enseignant est en charge d'offrir une structure analytique des œuvres étudiées. Le résultat est que les étudiants limitent leur utilisation de références au minimum requis pour satisfaire le remplissage de la page bibliographique, et n'ont pas reçu un enseignement pour une lecture critique de ces références, encore une fois tant sur le contenu que sur la forme.

La question suivante se présente ainsi : les carences en littéracie et en analyse métacognitive (et les conséquences "affectives » et attitudinales) sont-elles un héritage d'une approche à la transmission du savoir centrée sur le contenu et sur une relation unilatérale et autoritaire entre enseignant-expert et apprenants-novices ? La philosophie est peut-être le seul domaine qui, par sa nature, se prête d'emblée à un exercice inquisitoire par les apprenants -dans le but précisément, de développer une capacité à raisonner; mais en coexistence avec le principe selon lequel l'enseignant possède seul au final la «bonne» réponse. L'émergence récente d'une didactique centrée sur l'apprenant et favorisant autonomie et participation crée le contexte pédagogique nécessaire à une approche intégrative de la lecture et de l'écriture en langue seconde.

\subsection{Définir des objectifs}

Se référant aux «Instructions Officielles en vigueur au Portugal », Pereira (2004) note l'orientation de celles-ci envers l'écriture «non pas comme une simple résultante des connaissances sur la langue écrite (la grammaire) et de la lecture des textes, mais comme ayant un statut didactique à part entière ». Les objectifs définis par les Instructions Officielles du Portugal citées par Pereira méritent considération : « pratiquer l'écriture comme moyen de développer la compréhension dans la lecture ; produire des textes avec des intentions communicatives diversifiées; développer la conscience des différents modèles d'écrit; utiliser des techniques d'auto- et d'hétéro-correction; promouvoir la divulgation des écrits, en donnant un sens à la production. » (2004 : 321 ; texte original en anglais). Ces instructions qui portent sur le développement de compétences dans la langue natale semblent non moins pertinentes pour une langue seconde. Elles mettent clairement en avant la valeur pédagogique et (méta)cognitive de l'écriture non comme produit mais comme processus. La littéracie comme forme d'activité métalinguistique soutenue par Olson (1991) correspond à l'extériorisation de la cognition prônée par Sharples et Pemberton (1990). Dans les deux cas, il s'agit d'utiliser l'écriture comme un moyen d'activer une acquisition consciente et dynamique des connaissances. Le partage de la cognition (Legros \& Crinon, 2002) repose sur le même principe : c'est une autre forme d'extériorisation des connaissances, qui permet une prise de conscience, une réflexion sur le contenu par la mise en forme même de ce contenu. 
Le focus de Kamler et Thompson sur les étudiants en doctorat traite le problème en amont, là où les conséquences de ces carences se font le plus sentir, mais la solution idéale se trouve en aval : les étudiants passent d'une écriture de type 'composition-exercice' (démonstration de capacités) à une écriture 'académique' (contribution à un savoir) de façon abrupte, quand ils commencent une spécialisation en langue étrangère (après 4 années universitaires!). Rares sont ceux qui ont eu ou saisi l'opportunité de faire un travail de recherche (honors thesis), qui représente la première véritable initiation à la recherche académique. L'attitude implicite semble être que la recherche académique n'a pas lieu d'être avant la spécialisation. En contraste, Blyth (2008) affirme dans son programme éditorial sa volonté d'encourager « a greater participation of junior scholars, including graduate students » (2008: ix), soutenant que « [a] mixed editorial team of graduate students and supervising professors not only will assure that future volumes include a greater diversity of viewpoints from a wider variety of stakeholders but also will help more graduate students learn the ins and outs of academic publishing. » (ibidem).

D'autre part, la méta-analyse des articles académiques est quasi inexistante. Le paradoxe est donc que les étudiants « gradués » lisent quantité de textes pour leurs cours, mais que cette lecture est le plus souvent limitée à une discussion du contenu -sans discuter la forme. L'attitude implicite est, comme Kamler et Thompson le soulignent, que les étudiants vont apprivoiser le style, la forme, le jargon -l'écriture académique par la seule exposition répétée aux articles de journaux académiques, une version de « input processing ». Dans le contexte de l'acquisition de la compétence d'écriture, Pereira (2004:327) note aussi la présupposition (non fondée) par les enseignants du portugais (L1) aux niveaux primaire et secondaire d'un transfert automatique entre lecture de textes littéraires et la production de textes étudiants de qualité plus ou moins comparable («le slogan « lisez et vous écrirez » apparaît comme une certitude », 2004 : 327), transfert qui est également censé opérer d'un genre de texte (par exemple, la narration) à un autre (par exemple, le commentaire littéraire).

\section{5 Ébauche de méthode pour une littéracie et une métacognition en langue seconde}

\subsubsection{Rôle de l'enseignant, rôle de l'apprenant et modélisation}

Les mouvements « critiques » (pédagogie critique, littéracie critique, Critical Language Awareness) sont avant tout concernés par la contestation et ou la dénonciation de modèles dominants (Giroux, Bourdieu) et de la subjectivité de l'apprentissage déterminé par son contexte culturel, social, historique, et politique. Le rôle subjectif de l'enseignant est donc mis en question. Considérer la problématique politique n'est pas le but ici (dans quelle mesure l'enseignant impose une perspective, une idéologie, etc...), mais plutôt de re-évaluer le rôle participatif de l'apprenant dans une perspective d'accès plus direct au savoir. C'est cette acceptation du terme 'critique' à laquelle l'expression de 'Critical Language Learning' fait appel, tout au moins dans cette étude. Or reconsidérer la position de l'apprenant ne peut se faire sans reconsidérer celle de l'enseignant.

Dans quelle mesure peut-on assigner un rôle participatif à l'étudiant ? Dans cette optique, l'acquisition de connaissances s'inscrit dans une perspective de « conversations interactives, négociées » (Morgan, 1997 : 2). En d'autres termes, l'apprenant peut être un interlocuteur plutôt qu'un simple allocutaire. Cette perspective est particulièrement appropriée à la classe de langue étrangère, environnement dynamique dans lequel l'interaction est centrale. Le rôle de l'enseignant dans une structure pédagogique interactive doit être soigneusement réfléchi : il n'est ni désirable, ni possible d'abdiquer une "verticalité » des rapports enseignants-étudiants (Gale, 1996). Quelle que soit la méthode pédagogique adoptée, l'enseignant se trouve investi de l'autorité d'expert, de représentant de l'institution (académique), et d'une autorité personnelle (Gale, 1996 : 57). Little exprime la même idée lorsqu'il affirme que « les enseignants demeurent indispensables, en tant que pédagogues aussi bien qu'en tant qu'experts dans leur discipline. » (2007: 20). Kirschner et Sweller (2006) rejettent ainsi toutes les approches faisant usage d'une supervision minimale (« inquiry-based», « discovery-based», expérientielle, constructive) parce que, disent-ils, ces approches ne tiennent pas compte des rapports complexes entre les processus cognitifs de la 
mémoire à court et long terme. Entre autres, ils rappellent la différence qui existe entre apprendre et pratiquer une discipline, citant les travaux de Dehoney (1995) selon lequel les stratégies et modèles mentaux des experts sont le fruit d'un long processus d'accumulation d'expérience dans leur domaine (Kirschner \& Sweller, 2006 : 78).

Gale (1996) note aussi que l'étudiant manque de cette familiarité que possède l'enseignant-expert avec les normes, conventions, idéologies et thèmes du discours académique (1996: 80). Même si l'étudiant peut se conformer aux règles normatives d'écriture (académique), comme par exemple les règles structurelles, il ne peut créer un discours valable, par manque d'interaction avec le discours normatif. Selon Gale, les étudiants doivent donc être exposés aux textes «canoniques » au travers d'activités de lecture, d'écriture et de collaboration non dans le but de reproduire des textes normatifs mais pour littéralement obtenir voix au discours (1996: 91). Face à la dissociation qui existe dans les cours de composition anglaise (L1) entre les processus de lecture et d'écriture, un problème qui est selon elle largement ignoré par les enseignants, Gale demande ainsi : «Can we ask students to write an analytical paper without showing them what an analytical paper looks like or how people actually use these rules in their writing in different ways ? [...] Can we require them to revise and improve their writing without making them see the differences between good and poor texts through comparison and analysis?» (1996: 96). Pour Aulls (2002), c'est la modélisation par l'enseignant qui permet de créer un médium entre le contenu à acquérir et les procédures d'apprentissage. Cette modélisation s'applique aux procédures d'identification et de vérification de l'information, de reformulation de l'information (paraphrases), de l'utilisation de notes pour les activités de collaboration, et de la promotion d'un dialogue collaboratif.

S'appuyant sur les recherches de Petitjean (1998) en didactique de l'écriture, Pereira propose que «l'apprentissage de l'écriture nécessite d'être conduit par un enseignant qui connaît la dynamique et le processus de l'écriture, qui est capable d'en faire une transposition didactique convenable, mais aussi de prendre en compte le fait que l'élève, tout comme l'enseignant, est dépositaire d'un ensemble organisé de représentations sociales et cognitives, d'attitudes et de pratiques. » (2004: 320). Walsh explique par exemple que " [r]ather than simply 'handing over' to a group of learners by emphasizing pair- and groupwork, it is the teacher's ability to manage learner contributions which will, arguably, determine the success or otherwise of a lesson." (2006 : 3). Bien que ce commentaire soit dirigé vers l'interaction orale en classe de langue étrangère, il ne semble pas moins pertinent en ce qui concerne les activités écrites. Il s'agit donc d'engager l'étudiant dans des activités qui développent à la fois les connaissances sur le sujet et des aptitudes (méta)cognitives, c'est-à-dire d'exploiter la relation entre le processus d'apprentissage, le contenu qui est à acquérir, et l'usage que l'étudiant peut faire de cet acquis. En d'autres termes, il faut développer une approche intégrée dans laquelle la littéracie (lecture-analyse, écriture réflexive) et le contenu interagissent, chaque forme de connaissance en devenir servant le développement de l'autre.

\subsubsection{Quelques stratégies pratiques}

Les quelques stratégies présentées ici permettent d'envisager comment mettre en pratique les considérations précédentes. Elles ont notamment été systématiquement mises en œuvre dans deux cours sous-gradués avancés de français langue étrangère : langage et société, et le français moderne. En ellesmêmes, ces stratégies ne sont pas forcément nouvelles, et certaines sont très probablement familières aux enseignants. Ce qui diffère, c'est d'une part leur combinaison intentionnelle et d'autre part les objectifs qui leur sont assignés : engager l'apprenant à la fois sur le fond et sur la forme du texte ; faire le lien entre la lecture et l'écriture; envisager les différentes étapes (processus) dans la production d'un texte; explorer différents genres d'écrits; explorer le domaine de la recherche académique. L'ordre de présentation de ces stratégies réfléchit donc l'importance accordée au processus et à une approche intégrative de la littéracie et de la métacognition. La description des stratégies est renforcée par des illustrations concrètes issues des deux cours mentionnés, dans le cadre d'une approche collaborative et active à l'élaboration de connaissances centrée autour de la construction d'un wiki par les étudiants décrite dans Pellet (2010b). 
- Accompagner la lecture des étudiants : analysant les facteurs ayant abouti à la démotivation de ses étudiants (cours universitaire avancé de français sur le thème « le désir narratif »), Stivale (2004) note qu'ils auraient pu être moins désemparés et mieux préparés pour la lecture des textes si des questions plus ciblées et réfléchies les avaient orientés dans leur lecture. La méthode qui consiste à demander aux étudiants de lire le texte en préparation d'une discussion-analyse en classe ne demande qu'une lecture passive. L'apprenant dans cette perspective est facilement tenté de lire simplement pour connaître le thème et l'idée générale. C'est le problème d'invisibilité de la lecture soulevé par Macaro (2003). Une série de questions au fil du texte permet à l'apprenant de 'cibler' sa lecture, de faire attention aux éléments critiques, de ne pas 'se perdre en chemin'. Cela permet également de rendre l'exercice moins solitaire. L'étudiant n'est plus seul face au texte, il a avec lui les questions-guides, une sorte de plan de route. L'illustration pratique choisie provient du cours 'Langage et Société' discutant les notions de norme et d'attitudes. Les étudiants devaient lire pour ce cours un texte sur la variété linguistique (Klinkenberg, 1999), spécifiquement les sections 'Langue et stratification sociale : les attitudes' et 'Le problème de la norme' (pp. 49-54). Les étudiants travaillant en groupes devaient chacun préparer une réponse écrite à distribuer expliquer au reste de leur groupe. Les questions suivantes développées par l'enseignant ont servi de guide à la lecture ce texte : «Expliquez les notions suivantes: les deux types de corrélation en sociolinguistique; la notion de discours épilinguistique. Comment comprenez-vous «la guerre du nénufar » (vérifiez un dictionnaire...) ? Pourquoi parle-t-on de l'autonomie de la langue écrite? Expliquez la phrase (p.51 en haut) «la variation linguistique peut être mesurée objectivement par des spécialistes [mais] elle est aussi ressentie subjectivement. »; Expliquez norme objective $\sim$ norme évaluative et donnez des exemples de chaque dans la langue anglaise; Quels sont les cinq critères qui sont associés au 'bon usage'? Expliquez la phrase (p.52) "Dans tous les cas, la norme (évaluative) est toujours décrite comme correspondant à la norme objective d'un groupe donné. »; Qui est Vaugelas? Faites une petite recherche internet et écrivez 2-3 phrases avec vos propres mots ; pourquoi est-il important par rapport à la langue française; citez vos sources). Le dernier paragraphe p.52-53 est important: soyez capable d'expliquer l'idée essentielle à votre partenaire. Pour finir, qu'est-ce que "l'insécurité linguistique »? " Le bénéfice de ces questions est d'encourager les étudiants à une lecture active, orientée vers des objectifs pratiques et identifiés. Elles responsabilisent l'étudiant en lui donnant «un point d'arrivée » et un résultat tangible de leur effort intellectuel sous la forme de notes réfléchies. La discussion en classe prend davantage de sens et peut avoir lieu en groupes plutôt qu'être entièrement dominée par l'enseignant.

- La méta-analyse d'articles permet également de faire le lien entre lecture et écriture et d'approcher un texte sous un autre angle, mettant en évidence des aspects structurels ainsi que les stratégies textuelles mises en œuvre par l'auteur. Parce que les étudiants manquent souvent d'expérience à cet égard, la méta-analyse est une activité qui doit être modelée par l'enseignant (ce qui ne l'empêche pas de poser des questions). La méta-analyse est sans doute plus efficace si les étudiants ont déjà lu le texte pour le contenu qui y est présenté. Revenir sur un texte déjà 'présenté' en classe permet aussi de réactiver des connaissances d'une façon fraîche. Par la suite, cette activité peut être pratiquée en groupes (avec, comme pour l'activité précédente, une discussion dirigée par l'enseignant et qui sert à réaffirmer les commentaires en groupes). Bien que l'article choisi (Fleischman, 1997) pour une méta-analyse soit en anglais, les aspects évidents de cohérence structurelle et d'orientation pour le bénéfice du lecteur permettent une modélisation idéale. Rappelons que l'objectif métalangagier est de sensibiliser l'étudiant et de développer sa prise de conscience des traits de la langue qui sont explicitement orientés vers le lecteur: c'est-à-dire faciliter la lecture de l'article et par tant gagner sur le plan réthorique de l'argumentation. L'utilisation dans un pemier temps d'un texte L1 se trouve donc justifiée. Dans son article, Fleischman discute les résistances à la féminisation de la langue, s'attachant aux facteurs qui les soutiennent. Elle ouvre son article par deux exemples qu'elle contextualise ( $«$ Le capitaine Prieur est actuellement enceinte et l'accord prévoyait que dans ces circonstances, elle pouvait être rapatriée à Paris », p. 834 ; l'auteur Nicole Avril parle de sa nouvelle héroïne disant d'elle que « c'est un chirurgien car le mot chirurgienne n'existe pas officiellement » p. 835). Suite à cette introduction au sujet, Fleischman annonce explicitement l'objectif et l'argument 
soutenu («The basic question I propose to explore in this essay is... » «What I wish to suggest is that...» p. 835). Elle situe par ailleurs l'importance du sujet dans un contexte plus large : «This question is but one facet of a larger issue of why language change in general is so highly politicized in France » (p. 835). Fleischman termine son paragraphe avec le terme consacré 'bon usage', achevant ainsi de situer son sujet dans un contexte historique tout en utilisant une terminologie reconnue. À la fin de son introduction-contextualisation, Fleischman réitère l'objectif de son analyse sous forme de question («Why [do] language-users -including professional women themselves- seem to be so relecutant now to adopt these terms? »), rappelant qu'il s'agit d'une question cruciale, puis annonce la structure du reste de l'article (« The remainder of this essay will focus accordingly on the interplay of factors that might be invoked to account for French resistance to gender-based language reform. », p. 837). Cette modélisation détaillée se limite ici à l'introduction, mais en classe elle doit bien sûr porter sur l'ensemble du texte, et comprendre l'utilisation sélective des références et des citations; l'organisation des paragraphes (aspects structuraux de l'écriture), ou le positionnement stratégique d'assertions centrales (début ou fin de paragraphe par exemple). Les avantages de cette méta-analyse sont multiples : l'étudiant apprend à mieux lire, ou à lire de façon plus intelligente, critique; il apprend à identifier la sémiotique du texte. Cette acquisition permet une lecture plus active, engagée, et éventuellement critique, avec le contenu, le message du texte. Si le lien est fait par l'enseignant avec le projet écrit (étudiant) lié au cours, et si cette méta-analyse est répétée (avec des textes français), l'étudiant sera davantage en mesure de faire appel à ces modèles au moment de la rédaction du projet.

- Rédiger un abstract (un exercice académique) permet aussi d'activer la métacognition. Il suit logiquement la méta-analyse d'articles, et commence aussi par une modélisation préalable par l'enseignant. Une activité de comparaison d'abstracts rédigés individuellement par les étudiants permet à ceux-ci d'être plus objectifs, et donc plus critiques vis-à-vis de leur texte. Cette comparaison peut être rendue anonyme : l'enseignant recueille les travaux individuels et photocopie pour le cours suivant quelques uns en effaçant les noms de leurs auteurs. Cette approche permet une sélection par l'enseignant, de sorte de faire ressortir les qualités des abstracts plus réussis et de discuter les problèmes de ceux qui le sont moins (sans menacer la face de leurs auteurs). Le même bénéfice se retrouve que pour l'activité précédente : le processus même de rédaction du résumé force les étudiants à une lecture approfondie, à une analyse du contenu et de la force pour une rendition fidèle du texte original. Cette activité continue ainsi d'établir le lien entre lecture et écriture, encourageant les apprenants à entreprendre une lecture 'ciblée'. Faire choisir aux étudiants les trois meilleurs textes en leur demandant de justifier leur choix est une approche positive qui valorise les étudiants dont les textes sont choisis (sans que leurs auteurs se déclarent au reste de la classe) sans pour autant dévaloriser les autres 'auteurs'. En demandant aux étudiants de justifier leur(s) choix de façon spécifique les aspects constitutifs d'un abstract réussi force les étudiants à activement les identifier. Des exemples de résumés rédigés par les étudiants dans le cadre de leur projet de recherche final (cours Langage et Société) parlent d'eux-mêmes :

Titre : Le Parler Jeune : Une comparaison entre la langue de la jeunesse américaine et française

« Cet essaie discute le parler jeune aux Etats-Unis et en France. C'est un sujet important parce qu'on peut commence à comprendre quels traits du parler jeune dérivent de la jeunesse, ou bien de la langue soi-même. On commence par examiner les fonctionnes de l'argot, selon des auteurs français et américains. Par la suite, on examine la construction des mots argotiques, et on voit comment les médias et la technologie les ont influencées. Finalement, il y a une discussion des circonstances urbaines aux France et aux Etats-Unis, où on parle surtout de l'influence africaine au français. Aux Etats Unis, on se concentre sur les ebonics et la situation linguistique des jeunes africains américains. A la fin, on conclue que les similarités entre le parler jeune des deux pays est le résultat des circonstances sociaux semblables. »

Titre: Neutraliser les attitudes négatives envers le français du Québec: le promet dans l'enseignement 
«Cet article commence avec un court resume (pas compréhensif) de l'histoire linguistique au Québec, en mommant des différences entre le français parisien et le français du Québec. L'auteur écrit les attitudes négatives vers le «joual» du Québec. Elle explore les possibilités pour les préjugés contre la langue du Québec et le danger qui existe si ces préjugés sont renforcés. L'auteur discute des pensées différents concerant l'enseignement du français québecois dans la salle de classe et propose des idées pour faciliter une connaissance de l'histoire riche du français-canadien, une connaissance qui peut renverser ces attitudes négatifs et contribuer à la diversité de la plan linguistique dans les pays francophones. »

Titre : Diam's -une femme sur la scène d'une France en évolution

«Ce papier essaie d'explorer l'évolution sociale de la France par rapport aux messages de la rappeuse Diam's, une jeune femme immigrée des banlieues. De nos jours, la mondialisation, l'urbanisation et l'immigration avaient créé une population subordonnée qui a des tensions tangibles dans la société. Cette population est les «jeunes des banlieues» dont les gens et les agents du contrôle social stigmatisent. Comme une femme, Diam's s'affirme comme «un des garçons» en employant du langage brut et agressif qui la permet d'être vue comme une force dont il faut tenir compte. En plus, la musique rap ont des enjeux sociolinguistiques très importants surtout dans une nouvelle France très diverse culturellement grâce à l'immigration des anciens colons. Donc, la musique rap en France, malgré sa réputation comme un genre menacé par ses propres excès de la violence, des bandes et de l'insécurité, a vraiment la capacité de toucher une jeunesse en crise qui est justement difficile à accéder. Dans un analyse sociolinguistique de la chanson de Diam's, « Marine », la rappeuse fait un argument contre Marine Le Pen, un des chefs du Front National. Le message de cette chanson exprime la colère de l'artiste sur la discrimination des jeunes immigrés et sert comme un appel à gagner du respect pour cette nouvelle génération puissante. »

S'il est facile de voir les erreurs et les faiblesses (les fautes tendent à sauter aux yeux), il est cependant plus intéressant de regarder les traits démontrant qu'une réflexion et qu'une acquisition, même partielles, ont eu lieu. Au-delà des accents manquants, ce qui est plutôt frappant, ce sont les tentatives et un certain succès à rendre un 'ton' académique : annonce du sujet dans les trois cas; plan de l'essai ; impersonnalisation de la voix ('on', 'l'auteur', et effacement total de la voix dans le troisième exemple); et mise en avant de la signification de la recherche, directement («C'est un sujet important parce que...»), ou indirectement (« contribuer à la diversité de la plan linguistique » et « la musique rap ont des enjeux sociolinguistiques très importants »). Cet exercice encourage les étudiants à penser leur projet plus intégralement plutôt que comme une somme de sections successives, en même temps qu'il fournit une 'feuille de route' pour l'enseignant.

- Rédiger un projet de recherche ou d'analyse est fréquemment vécu comme une expérience solitaire par les auteurs, qu'ils soient étudiants ou non. C'est l'angoisse qui y est associée qui pousse certains à attendre la dernière minute pour 'pondre' un essai. En réponse à cette situation, une première suggestion est d'instaurer des étapes avec un calendrier prédéterminé encourageant les étudiants à construire leur projet plus rigoureusement et sur une plus longue échéance. Les étapes comprennent : le choix d'un sujet (soumis à l'approbation de l'enseignant) accompagné de quelques références bibliographiques (lesquelles constituent une sorte de garantie sur la viabilité du sujet); soumission d'un plan de travail incluant un objectif analytique (sous forme de question ou d'hypothèse) accompagnée d'une bibliographie plus élaborée; soumission du projet (qui peut éventuellement être retravaillé). Cette série d'étapes permet à l'enseignant d'offrir des commentaires (sur le fond et la forme en fonction des demandes liées au cours) qui peuvent réellement être pris en compte par les apprenants. Idéalement, l'apprenant a la possibilité de rencontrer l'enseignant si une clarification des commentaires ou une orientation supplémentaire sont nécessaires. Ces étapes créent autant de points d'échanges supplémentaires entre enseignant et apprenant, le premier étant alors en mesure d'orienter la réflexion et de pousser l'étudiant à un effort intellectuel et pratique supérieur à ce qu'il aurait été sans intervention et soutien. Par ailleurs, une session de discussion sur la notion de recherche permet de clarifier les objectifs en ce qu'ils sont en partie définis par un domaine et en partie par la vision particulière d'un enseignant. Une session de discussion amorce également le processus de réflexion 
des étudiants sur le sujet, la forme, et les actions nécessaires. Bien que des directives écrites soient certainement indispensables, ces directives risquent d'être davantage orientées vers les aspects (contraintes) pratiques (nombre de pages, etc...) ou vers un contenu limité à la thématique du projet. La signification profonde, intellectuelle du projet de recherche risque d'être incomprise ou ignorée par les étudiants. Une discussion de ce type est importante pour corriger des décalages entre croyances des étudiants et présomptions des enseignants sur ce que le projet implique ou même les objectifs.

- Un projet écrit collaboratif est une autre suggestion pour briser l'isolation liée à l'écriture. Un projet collaboratif (à deux ou en petits groupes) offrent aux étudiants la possibilité de partager et stimuler leurs efforts intellectuels et d'approcher le processus de recherche, d'analyse et d'écriture de façon plus rigoureuse qu'individuellement. Stivale (2004) propose qu'en ce qui concerne l'introduction de critique théorique dans les cours de littérature française, « less is more », c'est-à-dire que les étudiants risquent de perdre de vue la richesse du texte littéraire noyé dans des analyses théoriques trop nombreuses ou trop lourdes. De la même façon, le projet collaboratif qui peut apparaître à certains comme une moindre demande intellectuelle au premier abord, ne l'est pas si l'on considère le projet en tant que processus plutôt que produit. Il est probable qu'il favorise davantage de réflexion métacognitive que dans le cadre habituel du travail individuel. Ce partage de la cognition (Legros \& Crinon, 2002) se situe donc au double niveau du fond et de la forme. Une évaluation réciproque sur la qualité de la participation de chaque apprenant permet de plus d'établir une relative assurance d'intégrité. Les projets collaboratifs associés aux cours mentionnés sont particuliers dans la mesure où ils ont été produits sur une plateforme internet. L'objectif de ces projets était d'offrir aux étudiants un moyen de co-construire (en français) leur savoir sur chacun des sujets à travers un format authentique (le wiki, un site internet à but informatif développé par plusieurs participants-auteurs, c.f. Wikipedia). L'authenticité de ce format vient du fait qu'il n'est pas réservé au professeur, mais à la communauté constituée par les étudiants d'une part, et au public internet d'autre part. Les étudiants (en groupes) ont construit les deux sites wiki en directe correspondance avec les sujets abordés en classe et les textes lus pour le cours. La construction continue des wikis leur a permis de réfléchir sur les concepts et les textes (métacognition) pour décider de leur représentation, une sorte de percolation mentale. Le projet collaboratif peut donc permettre un engagement plus actif des étudiants avec le contenu du cours, au travers de l'écriture.

Bien d'autres stratégies peuvent être ajoutées à cette liste et trois autres sont abordées ici brièvement. Premièrement, les professeurs attendent des compétences méthodologiques qui ne correspondent pas nécessairement à la réalité. Une discussion sur la prise de notes sur un sujet particulier (en littérature, culture, ou concernant la langue) peut donc être profitable pour l'acquisition des connaissances dans ce domaine, ainsi que pour une approche plus critique d'autres textes. Cette discussion doit s'appuyer sur un article ou texte lu pour le cours, la discussion portant simultanément sur le contenu du texte et sur les notes que le texte lui-même doit générer. Une première étape consiste à provoquer une réflexion sur la prise de notes elle-même (objectif, étapes, et sélection d'informations) puis à proposer des consignes spécifiques. Entre autres, qu'est-ce que le titre évoque ? Y a-t-il un résumé déjà produit par l'auteur ? Quels sont les termes clés qui requièrent une définition? Quel est l'argument principal ? Quels exemples illustrent le mieux cet argument? Comment le mieux représenter (visuellement) et structurer les notes sur le papier pour assurer leur fonctionnalité ? Une deuxième étape consiste à modeler la prise de notes en appliquant les consignes à un texte lu par les étudiants. Trois 'règles du bon sens' font partie des consignes données aux étudiants des cours avancés : écrire ce que l'on comprend avec ses propres mots ; transformer en question ce qui n'est pas compris mais semble important; trouver un équilibre entre quantité et qualité. Comme c'est le cas avec les questions sur le texte, la discussion sur la prise de notes constitue donc une autre façon de guider la lecture des étudiants, en leur donnant cependant plus de responsabilité et d'autonomie.

Deuxièmement, le partage de connaissances entre étudiants travaillant sur des sujets similaires constitue un moyen d'approfondir la réflexion, d'envisager des perspectives différentes, et de partager des références. Les étudiants sont nécessairement amenés à comparer leur travail de recherche à celui des 
membres de leur groupe, ce qui peut leur suggérer des améliorations et contribuer à une plus grande motivation de fournir un effort intellectuel plutôt que de s'en tenir au premier jet.

Troisièmement, la présentation de textes en petits groupes force l'étudiant responsable d'un texte particulier à une lecture approfondie et critique, cet engagement actif avec le matériel se poursuivant dans la préparation de la présentation (sélection et synthèse du contenu ); tandis que les deux ou trois autres membres du groupe peuvent plus difficilement maintenir un rôle d'écoute passive (ce qui risque d'être le cas lorsque le professeur présente, ou lorsqu'un étudiant présente à toute la classe). L'utilisation de PowerPoint par les étudiants cherchait à remédier à l'effet soporifique, mais les visuels et les synthèses d'information qui y étaient associés se font que retarder (de quelques minutes ?) l'échéance de la déconnection mentale. Une présentation 'en petit comité' crée un plus grand équilibre entre rôle du présentateur et celui des autres participants au-delà du simple rôle d'auditeur.

Toutes ces stratégies impliquent la participation active de l'étudiant, mais également un rôle critique de l'enseignant qu'il s'agisse de la modélisation, ou de la modération de discussion. L'étudiant apprend à être autonome, mais, on peut le souhaiter, avec une anxiété et une isolation moindres que dans les situations évoquées par Kamler et Thompson (2004).

Ces stratégies peuvent apparaître évidentes ou même simplistes -et d'une certaine manière, elles le sont. Leur but est justement de mettre en évidence ces aspects de la langue qui sautent aux yeux des enseignants, mais demeurent parfois invisibles aux étudiants. L'enseignant possède une affinité et une familiarité avec le texte (qu'il soit lu ou qu'il soit écrit) que l'étudiant ne possède pas, et c'est cette expérience que l'enseignant doit partager, et pas seulement un savoir sur un objet particulier. Interrogés au cours et au terme du semestre, certains étudiants ont commenté que c'était la première fois qu'ils étaient amenés à écrire des 'abstracts' ou à réfléchir non seulement sur le contenu mais sur la forme des textes lus pour le cours. C'était peut-être la première fois parce que les textes comme les abstracts sont des écrits 'professionnels' réservés à des spécialistes. Cette explication avancée reflète l'un des problèmes essentiels des écrits étudiants, à savoir le fait qu'étant écrits 'juste pour le prof', ils ne sont pas authentiques. Le partage des connaissances, les approches collaboratives permettent de créer un lectorat ou un auditoire plus réel pour les étudiants.

Du point de vue de l'enseignant, ces stratégies ne requièrent pas tant une préparation supplémentaire pour le cours, qu'une préparation plus anticipée : par exemple, au lieu de préparer des questions (ou des points) de discussion sur un texte pour le jour où le texte est discuté, ces questions sont préparées au moment où l'enseignant demande à ses étudiants de lire le texte. Ces activités supportent une pédagogie dans laquelle la littéracie, un ensemble d'outils d'apprentissage réféchi liés à l'écriture et à la lecture, soutiennent le développement de connaissances, dans le cas présent, celles en français langue étrangère.

\section{Vers une pédagogie intégrative : l'exemple des nouvelles littéracies}

L'internet et le rôle de la communication médiatisée par ordinateur (CMO) dans la didactique des langues étrangères cristallisent les tendances évoquées jusqu'à présent. Les «nouvelles littéracies» (Coiro, Knobel, Lankshear \& Leu, 2008) commencent à être plus systématiquement exploitées par les enseignants de langues étrangères, tant dans l'acquisition de la langue (Thomas, 2009) que dans la formation pédagogique (Mangenot, 2004). L'objectif de ces nouvelles littéracies est de développer une capacité continue d'adaptation à de nouvelles formes de communication tout en reconnaissant qu' «il n'est plus possible pour qui que ce soit de maîtriser toutes les technologies en littéracie disponibles sur l'internet» (Coiro et al., 2008: 5 ; traduit de l'anglais). Cela rend d'autant plus critique la nécessité de réfléchir sur les applications pédagogiques qu'elles rendent possibles (Thorne, 2008). Le développement des activités pédagogiques plaçant l'écriture sur une plateforme internet (tels que blogs et wikis) correspond directement aux déficiences des activités écrites traditionnelles : activité écrite 'seulement' pour le professeur manquant d'authenticité, faisant de l'écrit un exercice rhétorique sans véritable lectorat ; texte-produit ; pédagogie souvent orientée sur la révision surtout de la forme. 
Ce que les nouvelles littéracies mettent aussi en avant, c'est la perception d'urgence de maîtriser les pratiques textuelles. L'impact de l'internet et de la CMO sur la nature de la litéracie constitue, selon Coiro et al., « une question de plus en plus urgente pour la recherche en éducation» (2008:1; traduit de l'anglais), les technologies de l'information évoluant et se répandant extrêmement rapidement ${ }^{2}$.

L'intersection des nouvelles littéracies avec les applications pédagogiques en langue seconde présente naturellement des traits communs au niveau du cadre théorique qui sous-tend le modèle pour une littéracie L2 critique proposé ici : les outils internet permettent en effet un apprentissage de la langue « in more dynamic, immediate, and communicative environments that provide opportunities for meaningful experiences through social constructivist learning » (Sturm, Kennell, McBride \& Kelly, 2009: 367), centré sur l'étudiant (plutôt que l'enseignant) et favorisant son autonomie. Ces traits communs suggèrent l'intérêt et la validité d'une comparaison pour tirer la leçon des succès mais peut-être davantage des difficultés des nouvelles littéracies L2 dans la réflexion sur le rôle de la littéracie 'traditionnelle' dans l'acquisition du français L2. Si la pédagogie liée à la littéracie par l'intermédiaire de la CMO présente des avantages clairs (ceux énoncés par Sturm et ses collègues ; voir aussi Warschauer, 2010), Kirkwood (entre autres) souligne qu'en termes de « e-learning : you don't always get what you hope for » (2009). Autrement dit, les outils que représente la CMO ne sont pas une solution miracle et demandent une exploitation intelligente de la part des enseignants en particulier vis-à-vis des attentes des étudiants. La question du rôle de l'enseignant, du 'feedback' offert à l'étudiant (qu'il s'agisse d'un 'acte' individuel ou collaboratif), et de l'évaluation sont trois aspects qui sont fréquemment soulignés comme définissant le succès ou l'échec de l'évènement pédagogique en ligne (Pellet, 2010b) -ces mêmes trois aspects qui conduisent à un désengagement des étudiants par rapport à leurs activités écrites L2 typiques (section 3.3). Le succès d'une pédagogie de la lecture et de l'écriture, quels que soient ses objectifs, dépend donc du degré d'interaction qui existe entre texte lu ou produit, enseignant et apprenant -mais rendent en même temps le processus d'évaluation plus difficile. La pédagogie adoptée, aussi bien que le système d'évaluation, doivent être non seulement rendus clairs par l'enseignant, mais aussi acceptés par les étudiants.

\section{Conclusion}

La littéracie et la métacognition sont proposées comme moyens d'engager activement les apprenants avec la signification des textes (au sens large) et les intentions (explicites et implicites) de leurs auteurs dans leurs contextes socio-culturels de production. Cette dimension plus consciente, ou critique de l'étude des manifestations de la langue étrangère peut s'appliquer à la tripartie littérature, culture et langue, établissant ainsi des 'ponts' entre ces trois pôles traditionnels du cursus de langue seconde, et permettant d'initier les apprenants à la recherche académique.

Les activités décrites dans la section précédente établissent un lien entre lecture, écriture, et métacognition pour une littéracie avancée en langue étrangère. Cette littéracie seconde représente une image miroir des objectifs visés par les recherches des liens existants (transfert) entre la langue première et la langue seconde citées dans l'introduction: August et Shanahan (2006) et Koda et Zehler (2008) cherchent à établir comment et jusqu'à quel point les compétences en littéracie L1 peuvent expliquer les mêmes compétences en L2. Cependant, pourquoi ne pas envisager les bénéfices possibles d'un transfert inverse, c'est-à-dire de la langue seconde ou étrangère vers la langue natale ? Les compétences L2 explicitement orientées sur la littéracie et la métacognition, soutenues par et soutenant une approche pédagogique procédurale envers l'écriture et la lecture, peuvent avoir des effets bénéfiques dans le raffinement des compétences en L1. L'étude d'une langue étrangère conduit par essence l'étudiant à réfléchir sur la langue. Le développement de compétences langagières et linguistiques à travers littéracie et métacognition mettent simplement davantage l'accent sur la langue en tant qu'outil de réflexion et d'apprentissage, plutôt qu'un outil de communication seulement, ce qui a généralement été l'objectif de l'acquisition d'une langue étrangère. Étant donné le discours actuel sur l'importance d'une éducation répondant au contexte de globalisation et d'une société d'information, une approche critique à l'enseignement des langues étrangères trouve une justification supplémentaire en ce que les retombées pédagogiques peuvent potentiellement s'étendre aux autres disciplines. L'étude d'une langue étrangère 
dans le cadre scolaire représente donc un site privilégié pour étendre les objectifs didactiques de ce domaine au-delà d'objectifs d'ouverture cross-culturelle. La perspective proposée sur la nature des objectifs en langue étrangère ne se limite pas aux étudiants avancés, qu'il s'agisse de ceux qui se destinent à l'enseignement primaire et secondaire, ou de ceux qui veulent obtenir un diplôme au-delà des quatre années typiques. S'il est vrai que les activités présentées donnent (un premier) accès aux domaines de recherche en langue, une sorte de société (académique) sinon secrète du moins relativement exclusive, l'objectif plus large est d'exploiter le potentiel représenté par le rôle de la langue dans l'acquisition des connaissances en général. Cette perspective est donc au contraire inclusive, et s'adresse à tous les étudiants, quel que soient leurs propres objectifs, et quel que soit leur niveau de connaissances langagières. Les enseignants de langue étrangère sont ainsi en mesure d'attirer l'attention des étudiants sur ces aspects de la langue qui peuvent demeurer opaques dans les autres disciplines.

Le terme « littéracie » est de plus en plus employé dans le milieu éducatif anglophone en association avec d'autres concepts («assessment literacy», «cultural literacy», «new media literacies», «multiliteracy»). Cette utilisation accrue reflète la capacité du terme à activer des notions cognitives liées à tel ou tel domaine invoqué par le terme jumelé (une terminologie, une problématique spécifiques), en même temps qu'il dénote un manque de compétences expressives (ou textuelles) et conceptuelles sur le domaine en question. La littéracie permet ainsi de mettre en avant une conception du savoir au-delà de connaissances 'domaniales' afin d'inclure la capacité à analyser et à réfléchir (à travers lecture et écriture) sur ces connaissances afin de pouvoir agir de façon critique. En d'autres termes, la littéracie met en jeu la notion de métacognition pour une appropriation des pratiques discursives. La dépendance grandissante envers la «littéracie », loin d'être un hasard, correspond à un contexte d'enjeux économiques se répercutant sur le milieu éducatif, lequel doit répondre à ce contexte par une formation permettant l'action -une action informée et critique. À cet égard, la maîtrise des pratiques discursives académiques représente un enjeu capital, pour ceux qui se destinent à ce milieu professionnel comme pour ceux, nombreux, dont le succès professionnel sera lié à leurs compétences discursives. La littéracie et la métacognition telles qu'elles ont été discutées ici représentent donc une opportunité pour l'enseignement du français langue étrangère d'élargir ses objectifs didactiques pour une conception plus complète de la langue et pour un rôle universitaire plus significatif.

\section{Références bibliographiques}

Anderson, B., Carr, W., Lewis, C., Salvatori, M. \& Turnbull, M. (2007). Effective literacy practices in FSL: Making connections. Toronto, Ontario: Pearson.

August, D. \& Shanahan, T. (2006). Developing literacy in second-language learners : Report of the national literacy panel on language minority children and youth. Mahwah, NJ : Erlbaum.

Aulls, M. W. (2002). The contributions of co-occurring forms of classroom discourse and academic activities to curriculum events and instruction. Journal of Educational Psychology, 94, 520-538.

Barbazan, M. (2008). Principes d'une grammaire prédictive du discours (français langue étrangère et maternelle), in : J. Durand, B. Habert \& B. Laks (éds.), Congrès Mondial de Linguistique Française 2008, 383-396.

Barré-De Miniac, C., Brissaud, C., \& Rispail, M. (éds.). 2004. La littéracie : conceptions théoriques et pratiques d'enseignement de la lecture-écriture. Paris : L'Harmattan.

Blyth, C. (éd.). (2008). From thought to action : exploring beliefs and outcomes in the foreign language program. Issues in Language Program Directions Vol. 18 of the American Association of University Supervisors, Coordinators, and Directors of Foreign Language Programs (AAUSC) Boston, MA : Heinle.

Blyth, C. (éd.). (2002). The Sociolinguistics of foreign-language classrooms: Contributions of the native, near-native, and non-native speaker. Issues in Language Program Directions Vol. 13 of the American Association of University Supervisors, Coordinators, and Directors of Foreign Language Programs (AAUSC) Boston, MA : Heinle.

Boyd, F. Brock, C., \& Rozendal, M. (éds.). (2004). Multicultural and multilingual literacy and language : contexts and practices. New York: Guilford Press. 
Bruner, J. (1968). Toward a theory of instruction. Harvard : Harvard University Press.

Byrnes, H. \& Maxim, H. (éds.). (2003). Advanced foreign language learning : a challenge to college program. Issues in Language Program Directions Vol. 14 of the American Association of University Supervisors, Coordinators, and Directors of Foreign Language Programs (AAUSC) Boston, MA : Heinle.

Byrnes, H., \& Sprang, K. (2003). Fostering advanced L2 literacy : a genre-based, cognitive approach. In : Byrnes et Maxim (éds). 47-85.

Clarke, M. A. (1979). Reading in Spanish and English : evidence from adult ESL students. Language Learning, 29, $121-50$.

Colby, A., Ehrlich, T., Beaumont, E., \& Stephens, J. (2003). Educating citizens: Preparing America's undergraduates for lives of moral and civic responsibility. San Francisco: Jossey-Bass.

Cochinaux, P. \& de Woot, P. (1995). Moving towards a learning society. A CRE-ERT forum report on European education. Genève : CRE et Bruxelles : ERT.

Coiro, J., Knobel, M., Lankshear, C. \& Leu, D. (2008). Handbook of research on new literacies. New York : Erlbaum.

Crystal, D. (2001). Language and the internet. Cambridge : Cambridge University Press.

Dehoney, J. (1995). Cognitive task analysis: Implications for the theory and practice of instructional design. Proceedings of the Annual National Convention of the Association for Educational Communications and Technology (AECT), 113-123. (ERIC Document Reproduction Service No. ED 383 294).

Elalouf, M.-L. (2008). La reception des travaux en didactique de la langue : quelles résistances ? In : J. Durand, B. Habert \& B. Laks (éds.), Congrès Mondial de Linguistique Française 2008, 599-603.

Elola, I. (2008). Portrait of a teacher: beliefs on feedback and revision in the foreign language classroom. In : C. Blyth, (éd.) (2008).

Fairclough, N. (éd.) (1992). Critical language awareness. New York : Longman.

Fairclough, N. (2006). Language and globalization. New York : Routledge.

Farias, M. (2005). Critical language awareness in foreign language learning. Literatura y Lingüistica 16. 211-222.

Fleischman, S. (1997). The battle of feminism and bon usage : instituting nonsexist usage in French. The French Review 70, 6, 834-844.

Flower, L. \& Hayes, J.R. (1981). A cognitive process theory of writing. In : College Composition and Communication, 32, 365-387.

Gale, X.L. (1996). Teachers, discourses, and authority in the postmodern composition classroom. New York : State University of New York Press.

Gass, S., Bardovi-Harlig, K., Sieloff-Magnan, S., \& Walz, J. (2002). Pedagogical norms for second and foreign language learning and teaching: Studies in honour of Albert Valdman. Philadelphia : John Benjamins.

Jaffré, J.-P. (2004). La littéracie : histoire d'un mot, effets d'un concept. In : C. Barré-De Miniac, C. Brissaud \& M. Rispail (éds.), 21-41.

Kamler, B. \& Thompson, P. (2004). Driven to abstraction : doctoral supervision and writing pedagogy. Teaching in Higher Education 9,2, 195-209.

Katz, S. \& Blyth, C. (2007). Teaching French grammar in context. New Haven : Yale University Press.

Kern, R. (2003). Literacy and advanced foreign language learning: rethinking the curriculum. In : Byrnes et Maxim (éds), 2-18.

Kirschner, P., Sweller, J. and Clark, R. (2006). Why minimum guidance does not work: An analysis of the failure of Constructivist, Discovery, Problem-based, Experiential, and Inquiry-based learning. Educational Psychologist, 41.2, 75-86.

Klinkenberg, J.-M. (1999). Des langues romanes : introduction aux études de linguistique romanes. Paris : Duculot. 
Koda, K. \& Zehler, A. (éds.). (2008). Learning to read across languages: Cross-linguistic relationships in first-and second-language literacy development. New York : Routledge.

Kohonen, V. (1998). Authentic assessment as an integration of language learning, teaching, evaluation and the teacher's professional growth, in : A. Huhta, V. Kohonen, L. Kurki-Suonio, \& S. Luoma (éds) Current developments and alternatives in language assessments. Proceedings of LRTC 96, Jyväskylä : University of Jyväskylä, Center for Applied Language Studies. 7-22.

Kramsch, C. (1997). The Privilege of the Non-Native Speaker. In : PMLA, 112, 359-69.

Kumaravadivelu, B. (2002). Beyond methods: macrostrategies for language teaching. Yale : Yale University Press.

Leithwood, K., Jantzi, D. \& Steinbach, R. (1999). Changing leadership for changing times. Philadelphia: Open University Press.

Legros, D. \& Crinon, J. (2002). Psychologie des apprentissages et multimédia. Paris : Armand Colin université.

Lenart, A., Arafeh, S., Smith, A., \& Macgill, A. R. (2008). Writing, Technology and Teens. Washington, D.C. : Pew Internet \& American Life Project.

Little, D. (2007). Language learner autonomy: some fundamental considerations revisited. Innovation in Language Learning and Teaching, 1.1,14-29.

Lundwall, B.-A. (2000). Europe and the learning economy-on the need to for reintegrating strategies of firms, social partners and policy makers. Towards a learning society: Innovation and competence building with social cohesion for Europe. A seminar on socio-economic research and European policy. Lisbonne, 28-30 mai 2000.

Macaro, E. (2003). Teaching and learning a second language: A review of recent research. New York : Continuum.

Mangenot, F. (2004). Analyse sémio-pragmatique des forums pédagogiques, in J.-M. Salaün \& C. Vandendorpe, (éds.), Les défis de la publication sur le Web : hyperlectures, cybertextes et méta-éditions. Villeurbanne : Presses de l'Enssib. pp. 103-123.

Magnotto Neff, J. \& Whithaus, C. (2008). Writing across distances and disciplines: Research and pedagogy in distributed learning. New York : Erlbaum.

Maxim, H. (2003). Expanding visions for collegiate advanced foreign language learning. In : Byrnes et Maxim (éds.). 180-191.

Mondada, L. (2008). Contributions de la linguistique interactionnelle. In: J. Durand, B. Habert \& B. Laks (éds.), Congrès Mondial de Linguistique Française 2008, 881-897.

Morgan, W. (1997). Critical literacy in the classroom : the art of the possible. New York : Routledge.

New London Group. (1996). A pedagogy of multiliteracies : designing social futures. Harvard Educational Review $66(1), 60-92$.

Niemi, H. (2002). Active learning-A cultural change needed in teacher education and schools. Teaching and Teacher Education, 18, 763-780.

Olson, D.R. (1999). Literacy and language development. In : D.A. Wagner, R.L. Venezky, B.V. Street (éds.), Literacy. An international handbook. Boulder, CO: Westview Press, 132-136.

Pellet, S. (2010a). Focusing on empowerment: Higher Education participatory model and Critical Language Learning. AAUP Conference on the State of Higher Education, juin 2010.

Pellet, S. (2010b). Wikis for Building Content Knowledge in the Foreign Language Classroom. Recherche non publiée.

Petitjean, A. (1998). Enseignement / apprentissage de l'écriture et transposition didactique. Pratiques, 97/98, 105132.

Pereira, L. (2004). Des discours sur les pratiques aux pratiques d'enseignement. In : C. Barré-De Miniac, C. Brissaud \& M. Rispail (éds.), 319-332.

Russel, D. (2002). Writing in the academic disciplines : a curricular history. Carbondale : Southern Illinois University Press. 
Savignon, S. (2007). Beyond communicative language teaching: what's ahead? Journal of Pragmatics, 39, 207-220.

Sharples, M. \& Pemberton, L. (1990). Starting from the writer: guidelines for the design of user-centred document processors. Computer Assisted Language Learning, 1, 37-57. Oxford : Intellect.

Slevin, J. (2007). Academic literacy and the discipline of English. In: R. Feal (éd.), Profession 2007. New York : The Modern Language Association of America.

Stivale, C. (éd.) (2004). Modern French Literary Studies in the classroom : pedagogical strategies. New York: The Modern Language Association.

Swaffar, J. (2003). A template for advanced learner tasks : staging gender reading and cultural literacy through the précis. In : Byrnes et Maxim (éds), 19-45.

Swaffar, J. \& Arens, K. (2005). Remapping the foreign language curriculum: An approach through multiple literacies. New York: The Modern Language Association of America.

Terry, R. (1989). Teaching and evaluating writing as a communicative skill. Foreign Language Annals, 22, 43-53.

Thomas, M. (2009). Handbook of research on Web 2.0 and second language learning. Hershey: Information Science Reference.

Thorne, S. \& Reinhardt, J. (2008). "Bridging activities", New Media Literacies, and advanced foreign language proficiency. CALICO Journal 25 (3), 558-572.

Train, R. W. (2007). Language ideology and foreign language pedagogy. In D. Ayoun (éd.), French applied linguistics. Amsterdam : John Benjamins. 238-269.

Train, R. W. (2003). The (non)native standard language in foreign language education : A critical perspective. In C. Blyth (éd.), 2003. 3-39.

Valdès, G., Bunch, G., Snow, C., \& Lee, C. avec L. Matos. (2005). Enhancing the development of students' language(s). In : L. Darling-Hammond \& J. Bransford (éds.), Preparing teachers for a changing world: What teachers should learn and be able to do. San Francisco : Josey Bass.

Walsh, S. (2006). Investigating classroom discourse. New York : Routledge.

Ware, P. \& Kramsch, C. (2005). Toward an intercultural stance: Teaching German and English through tellecollaboration. The Modern Language Journal, 89, 189-205.

Warga, M. (2007). Interlanguage pragmatics in L2 French, in: D. Ayoun (éd.), French applied linguistics. Amsterdam : John Benjamins.

Way, D., Joiner, E.G., \& Seaman, M.A. (2000). Writing in the secondary foreign language classroom: the effects of prompts and tasks on novice learners of French. The Modern Language Journal, 84, 2, 171-84.

Weber-Fève, S. (2009). A Writing Design: Using Abstracts in the Writing Process. French Review, 82:6, 103-115.

White Paper on Education and Training. (1995). Bruxelles: Commission of the European Communities.

\footnotetext{
${ }^{1}$ Ce groupe comporte : Courtney Cazden, Bill Cope, Norman Fairclough, James Gee, Mary Kalantzis, Gunther Kress, Allen Luke, Carmen Luke, Sarah Michaels, et Martin Nakata.

${ }^{2}$ S'appuyant sur des statistiques sur l'usage de l'internet à travers le monde, Coiro et ses collègues estiment que «pratiquement la moitié de la population sera en ligne d'ici 2012, et que presque toute la population aura accès internet $\gg(2008: 3)$.
} 